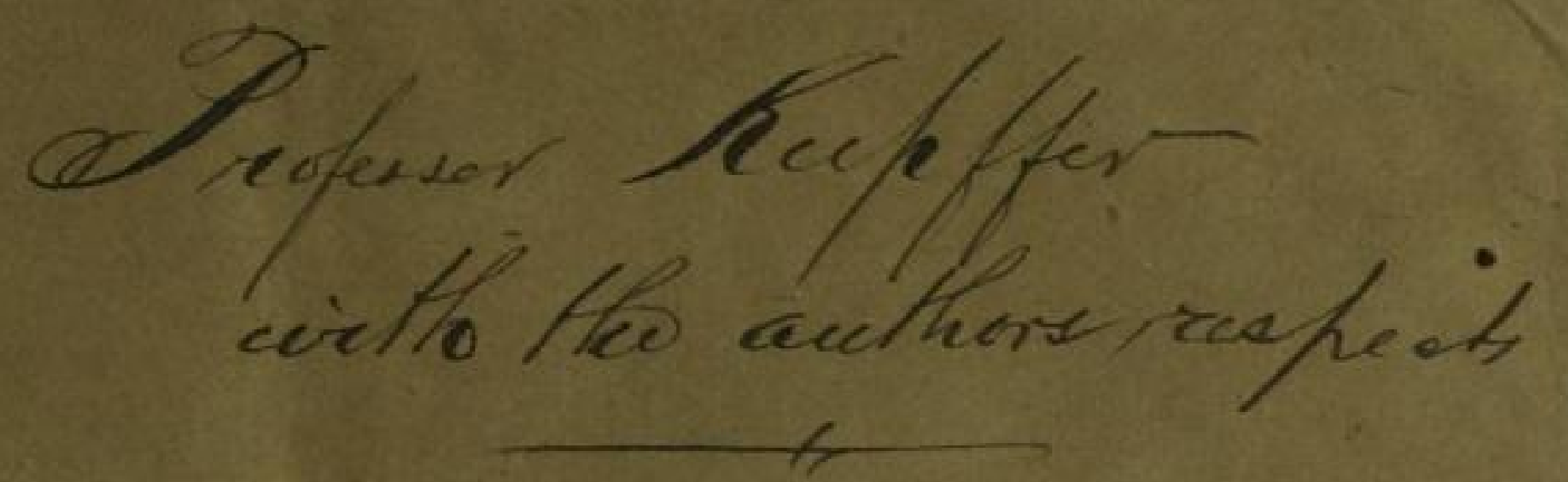

\title{
Physica. 305,50
}


$\$$ 


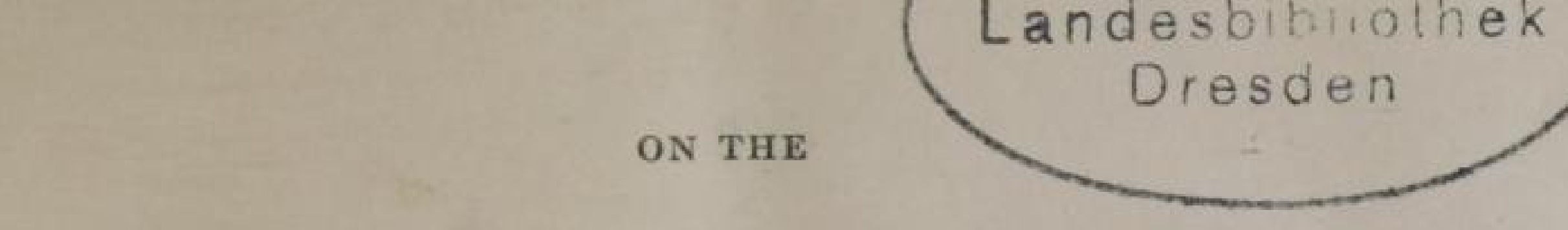

\section{THERMAL EFFECTS OF FLUIDS IN MOTION :}

TEMPERATURE OF A BODY MOVING SLOWLY

THROUGH AIR.

By Prof. W. THOMSON, F.R.S., and J. P. JOULE, Esq., F.R.S.

The motion of air in the neighbourhood of a body moving very slowly through it, may be approximately determined by treating the problem as if air were an incompressible fluid. The ordinary hydrodynamical equations, so applied, give the velocity and the pressure of the fluid at any point ; and the variations of density and temperature actually experienced by the air are approximately determined by using the approximate evaluation of the pressure thus obtained. Now, if a solid of any shape be carried uniformly through a perfect liquid*, it experiences fluid-pressure at different parts of its surface, expressed by the following formula,-

$$
p=\Pi+\frac{1}{2} \rho\left(\mathrm{V}^{2}-q^{2}\right),
$$

where II denotes the fluid-pressure at considerable distances from the solid, $\rho$ the mass of unity of volume of the fluid, $\mathrm{V}$ the velocity of translation of the solid, and $q$ the velocity of the fluid relatively to the solid, at the point of its surface in question. The effect of this pressure on the whole is, no resultant force, and only a resultant couple which vanishes in certain cases, including all in which the solid is symmetrical with reference to the direction of motion. If the surface of the body be everywhere convex, there will be an augmentation of pressure in the fore and after parts of it, and a diminution of pressure round a medium zone. There are clearly in every such case just two points of the surface of the solid, one in the fore part, and the other in the after part, at which the velocity of the fluid relatively to it is zero, and which we may call the fore and after pole respectively. The middle region round the body in which the relative velocity exceeds $\mathrm{V}$, and where consequently the fluid pressure is diminished by the motion, may be called the equatorial zone; and where there is a definite middle line, or line of maximum relative volocity, this line will be called the equator.

* That is, as we shall call it for brevity, an ideal fluid, perfectly incompressible and perfectly free from mutual friction among its parts. 
If the fluid be air instead of the ideal " perfect liquid," and if the motion be slow enough to admit of the approximation referred to above, there will be a heating effect on the fore and after parts of the body, and a cooling effect on the equatorial zone. If the dimensions and the thermal conductivity of the body be such that there is no sensible loss on these heating and cooling effects by conduction, the temperature maintained at any point of the surface by the air flowing against it, will be given by the equation

$$
t=\Theta\left(\frac{p}{\Pi}\right)^{\frac{4.41}{1 \cdot 41}}
$$

where $\theta$ denotes the temperature of the air as uninfluenced by the motion, and $p$ and $\Pi$ denote the same as before*. Hence, using for $p$ its value by the preceding equation, we have

$$
t=\theta\left\{1+\frac{\rho}{2 \Pi}\left(\mathrm{V}^{2}-q^{2}\right)\right\}^{\frac{41}{1 \cdot 41}}
$$

But if $\mathrm{H}$ denote the length of a column of homogeneous atmosphere, of which the weight is equal to the pressure on its perpendicular section, and if $g$ denote the dynamical measure of the force of gravity $(32 \cdot 2$ in feet per second of velocity generated per second), we have

$$
g_{\rho} \mathrm{H}=\mathrm{II} \text {; }
$$

and if we denote by $\alpha$ the velocity of sound in the air, which is equal to $\sqrt{1.41 \times g \mathrm{H}}$, the expression for the temperature becomes

$$
t=\theta\left\{1+\frac{1 \cdot 41}{2} \cdot \frac{V^{2}-q^{2}}{\alpha^{2}}\right\}^{\frac{-41}{1 \cdot 41}}
$$

According to the supposition on which our approximation depends, that the velocity of the motion is small, that is, as we now see, a small fraction of the velocity of sound, this expression becomes

$$
t=\theta\left\{1+\cdot 41 \times \frac{V^{2}-q^{2}}{2 \alpha^{2}}\right\} .
$$

At either the fore or after pole, or generally at every point where the velocity of the air relatively to the solid vanishes (at a re-entrant

* The temperatures are reckoned according to the absolute thermodynamic scale which we have proposed, and may, to a degree of accuracy correspondent with that of the ordinary " gaseous laws," be taken as temperature Centigrade by the air-thermometer, with $273^{\circ} \cdot 7$ added in each case. See the author's previous paper "On the Thermal Effects of Fluids in Motion," Part II., Philosophical Transactions, 1854 , part 2. p. 353 . 
angle for instance, if there is such), we have $q=0$, and therefore an elevation of temperature amounting to

$$
\cdot 41 \times \frac{V^{2}}{2 \alpha^{2}} \theta \text {. }
$$

If, for instance, the absolute temperature, $\theta$, of the air at a distance from the solid be $287^{\circ}$ (that is $55^{\circ}$ on the Fahr. scale), for which the velocity of sound is 1115 per second, the elevation of temperature at a pole, or at any point of no relative motion, will be, in degrees Centigrade,

$$
58^{\circ} .8 \times\left(\frac{\mathrm{V}}{\alpha}\right)^{2} \text {, or } 58^{\circ} \cdot 8 \times\left(\frac{\mathrm{V}}{1115}\right)^{2},
$$

the velocity $\mathrm{V}$ being reckoned in feet per second. If, for instance, the velocity of the body through the air be 88 feet per second ( 60 miles an hour), the elevation of temperature at the points of no relative motion is $\cdot 36^{\circ}$, or rather more than $\frac{1}{3}$ of a degree Centigrade.

To find the greatest depression of temperature in any case, it is necessary to take the form of the body into account. If this be spherical, the absolute velocity of the fluid backwards across the equator will be half the velocity of the ball forwards; or the relative velocity $(q)$ of the fluid across the equator will be $\frac{3}{2}$ of the velocity of the solid. Hence the depression of temperature at the equator of a sphere moving slowly through the air will be just $\frac{9}{4}$ of the elevation of temperature at each pole. It is obvious from this that a spheroid of revolution, moving in the direction of its axis, would experience at its equator a depression of temperature, greater if it be an oblate spheroid, or less if it be a prolate spheroid, than $\frac{9}{4}$ of the elevation of temperature at each pole.

It must be borne in mind, that, besides the limitation to velocities of the body small in comparison with the velocity of sound, these conclusions involve the supposition that the relative motions of the different parts of the air are unresisted by mutual friction, a supposition which is not even approximately true in most cases that can come under observation. Even in the case of a ball pendulum vibrating in air, Professor Stokes* finds that the motion is seriously influenced

* "On the Effect of the Internal Friction of Fluids on the Motion of Pendulums," read to the Cambridge Philosophical Society, Dec. 9, 1850, and published in vol. ix. part 2 of their Transactions. 
by fluid friction. Hence with velocities which could give any effect sensible on even the most delicate of the ether thermometers yet made (330 divisions to a degree), it is not to be expected that anything like a complete verification or even illustration of the preceding theory, involving the assumption of no friction, can be had. It is probable that the forward polar region of heating effect will, in consequence of fluid friction, become gradually larger as the velocity is increased, until it spreads over the whole equatorial region, and does away with all cooling effects.

Our experimental inquiry has hitherto been chiefly directed to ascertain the law of the thermal effect upon a thermometer rapidly whirled in the air. We have also made some experiments on the modifying effects of resisting envelopes, and on the temperatures at different parts of the surface of a whirled globe. The whirling apparatus consisted of a wheel worked by haind, communicating rapid rotation to an axle, at the extremity of which an arm carrying the thermometer with its bulb outwards was fixed. The distance between the centre of the axle and the thermometer bulb was in all the experiments 39 inches. The thermometers made use of were filled with ether or chloroform, and had, the smaller 275 , and the larger 330 divisions to the degree Centigrade. The lengths of the cylindrical bulbs were $\frac{9}{10}$ and $1 \frac{4}{10}$ inch, their diameters $\cdot 26$ and $\cdot 48$ of an inch respectively.

TABLE I.-Small bulb Thermometer.

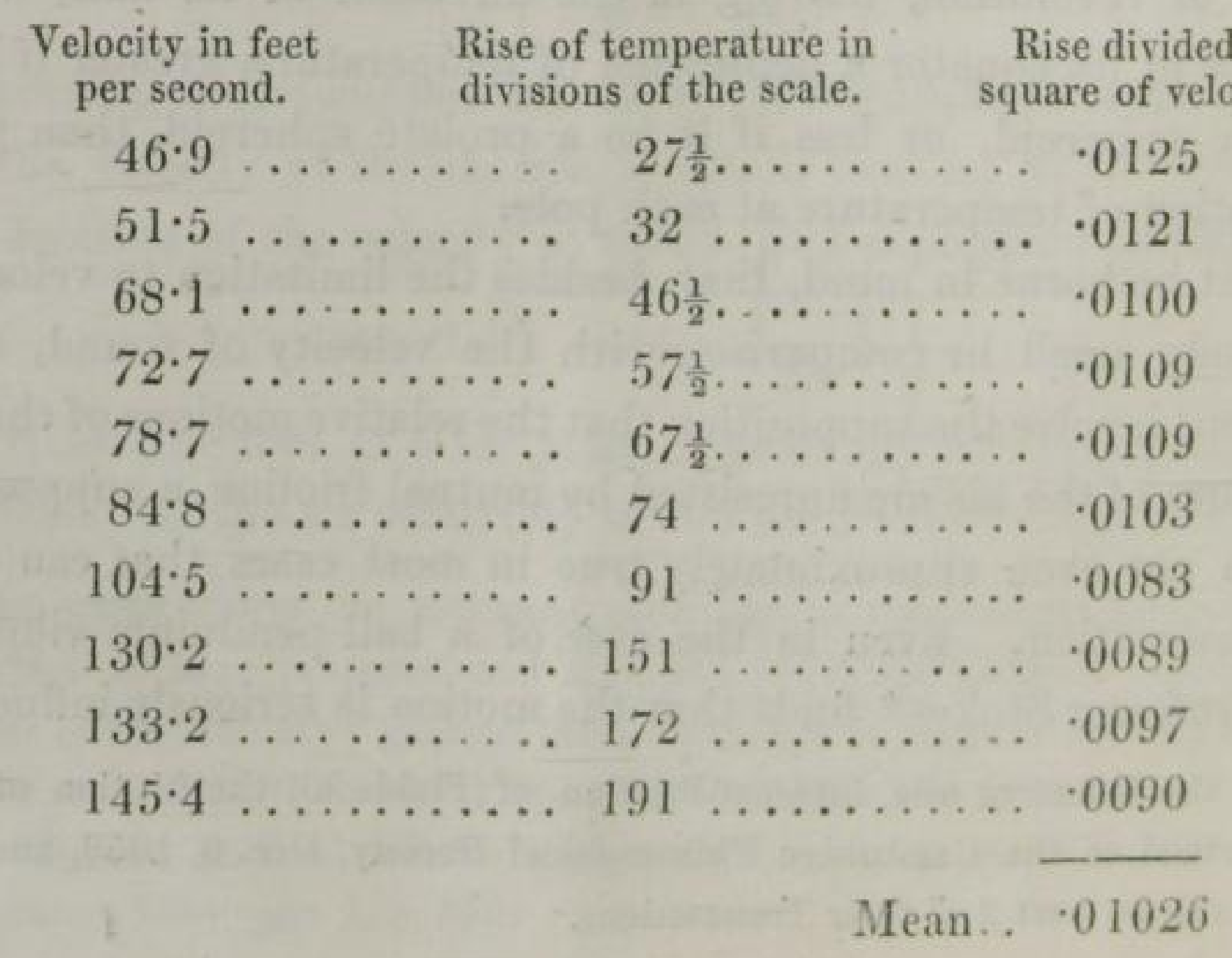


The above Table shows an increase of temperature nearly proportional to the square of the velocity.

$$
V=\sqrt{\frac{275}{01026}}=163 \cdot 7=\text { the velocity in feet per second, which, }
$$

in air of the same density, would have raised the temperature $1^{\circ}$ Centigrade.

TABLE II.-Larger bulb Thermometer.

$\begin{array}{ccc}\begin{array}{c}\text { Velocity in feet } \\ \text { per second. }\end{array} & \begin{array}{c}\text { Rise of temperature in } \\ \text { divisions of scale. }\end{array} & \begin{array}{c}\text { Rise divided by } \\ \text { square of velocity. }\end{array}\end{array}$

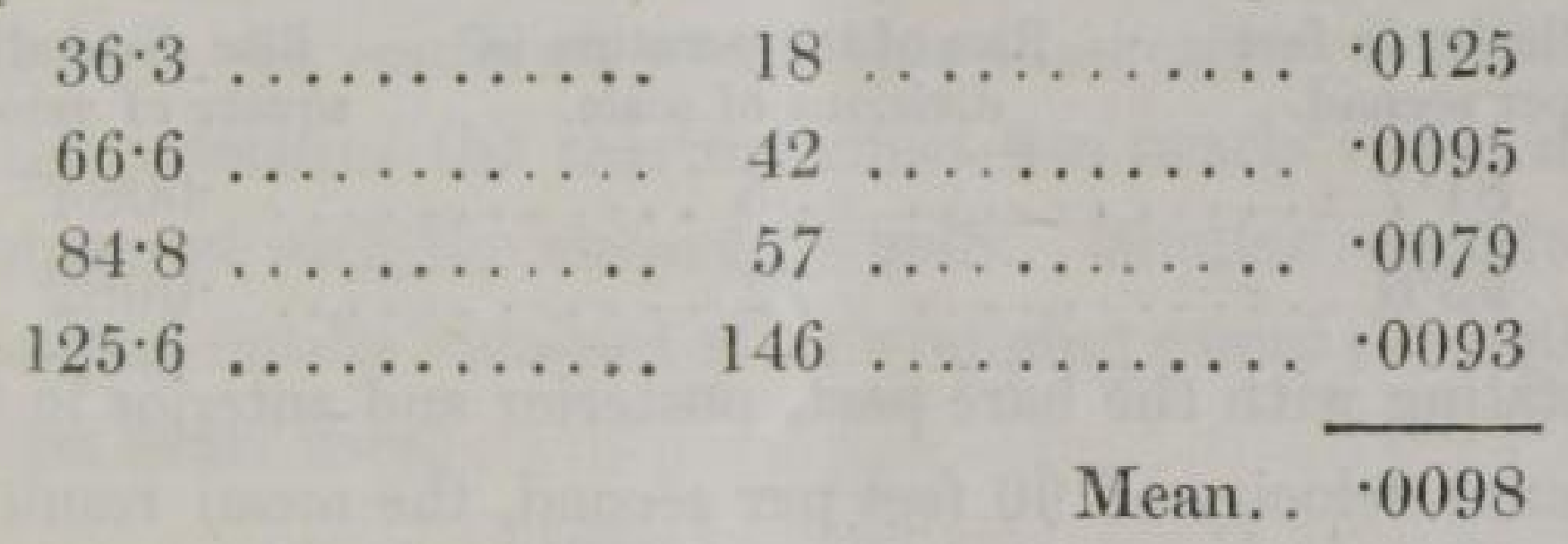

In this instance $\mathrm{V}=\sqrt{\frac{330}{\cdot 0098}}=183.5$ feet per second for $1^{\circ} \mathrm{Cen}$ tigrade. It is however possible that the full thermal effect was not so completely attained in three minutes (the time occupied by each whirling) as with the smaller bulb. On the whole it did not appear to us that the experiments justified the conclusion, that an increase of the dimensions of the bulb was accompanied by an alteration of the thermal effect.

TABLE III.-Larger bulb Thermometer covered with five folds of writing-paper.

\begin{tabular}{|c|c|c|}
\hline $\begin{array}{l}\text { Velocity in feet } \\
\text { per second. }\end{array}$ & $\begin{array}{l}\text { Rise of temperature in } \\
\text { divisions of scale. }\end{array}$ & $\begin{array}{l}\text { Rise divided } \\
\text { square of vel }\end{array}$ \\
\hline $36 \cdot 3 \ldots$ & $\ldots \ldots \quad 20 \ldots \ldots$ & $\ldots \cdot 0152$ \\
\hline $51 \cdot 5$ & 43 . & .. $\cdot 0162$ \\
\hline $72 \cdot 6 \ldots$ & $\ldots \ldots 53 \ldots$ & $\ldots \cdot 0101$ \\
\hline $118 \quad \ldots$ & ... 132 . & .. $\cdot 009$ \\
\hline
\end{tabular}

The increased thermal effect at comparatively slow velocities, exhibited in the above Table, appeared to be owing to the friction of the air against the paper surface being greater than against the polished glass surface.

One quarter of the enveloping paper was now removed, and the bulb whirled with its bared part in the rear. The results were as follow :- 
TABLE IV.-Paper removed from posterior side.

\begin{tabular}{c}
$\begin{array}{c}\text { Velocity in feet } \\
\text { per second. }\end{array}$ \\
$\begin{array}{c}75 \cdot 6 \ldots \ldots \ldots \ldots \\
96 \cdot 8 \ldots \ldots \ldots \ldots\end{array} \quad \begin{array}{c}\text { Rise of temperature in } \\
\text { divisions of scale. }\end{array} \quad \begin{array}{c}\text { Rise divided by } \\
\text { square of velocity. }\end{array}$ \\
\hline
\end{tabular}

On whirling in the contrary direction, so that the naked part of the bulb went first, we got,-

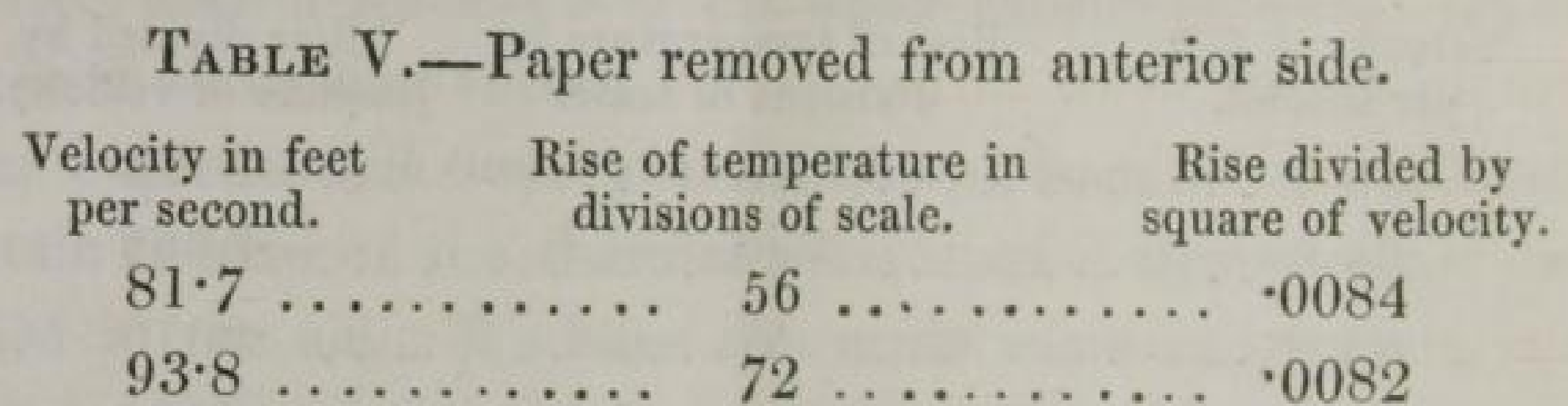

On rotating with the bare part, posterior and anterior in turns, at the constant velocity of 90 feet per second, the mean result did not appear to indicate any decided difference of thermal effect.

Another quarter of paper was now removed from the opposite side. Then on whirling so that the bared parts were anterior and posterior, we obtained a rise of 83 divisions with a velocity of 93.8 . But on turning the thermometer on its axis one quarter round, so that the bared parts were on each side, we found the somewhat smaller rise of 62 divisions for a velocity of 90.8 feet per second.

The effect of surface friction haring been exhibited at slow velocities with the papered bulb, we were induced to try the effect of increasing it by wrapping iron wire round the bulb.

TABLE VI.-Larger bulb Thermometer wrapped with iron wire.

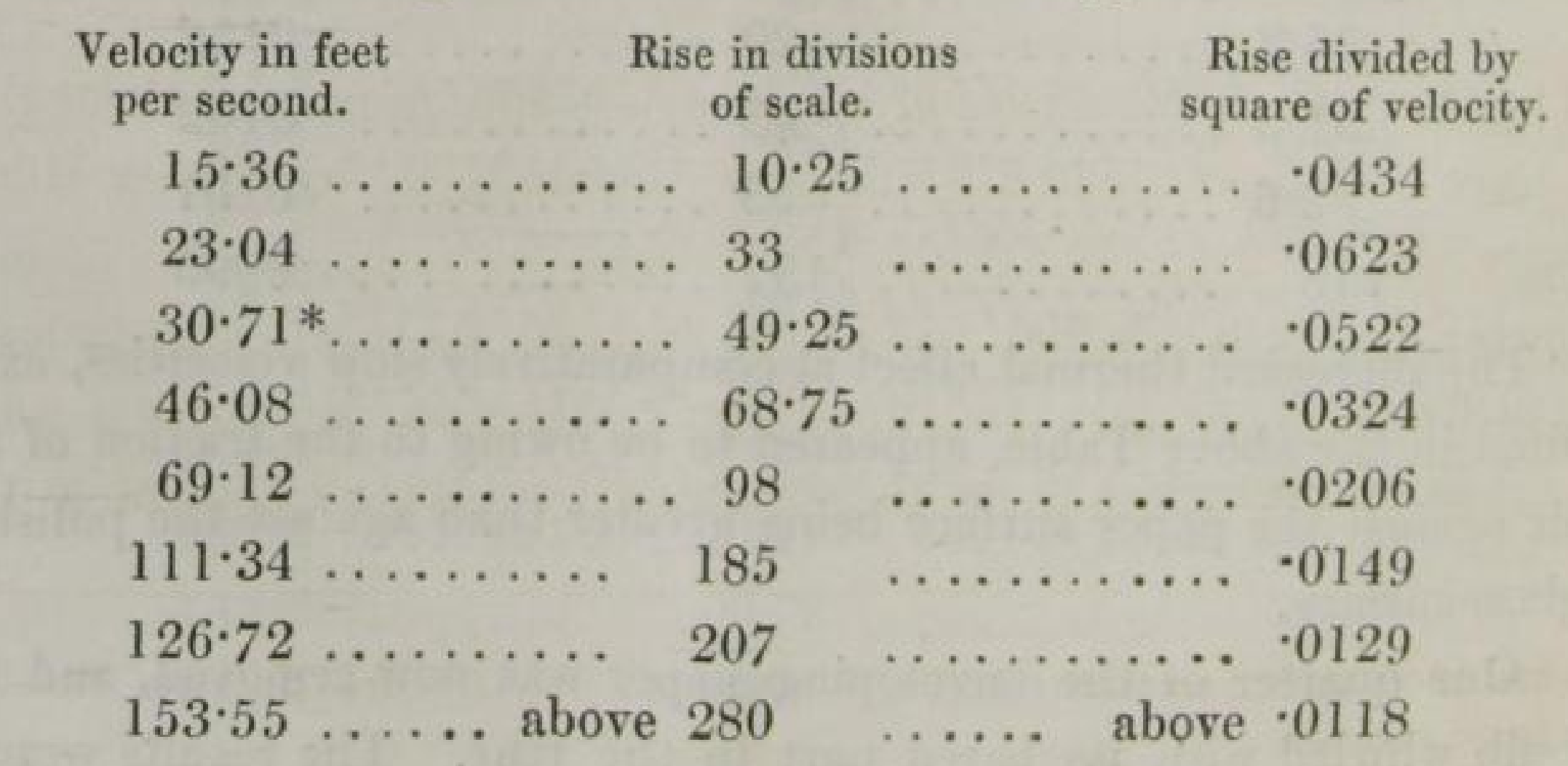

* The whirring sound began at this velocity. According to its intensity the 
On inspecting the above Table, it will be seen that the thermal effect produced at slow velocities was five times as great as with the bare bulb. This increase is evidently due to friction. In fact, as one layer of wire was employed, and the coils were not so close as to prevent the access of air between them, the surface must have been about four times as great as that of the uncovered bulb. At high velocities, it is probable that a cushion of air which has not time to escape past resisting obstacles makes the actual friction almost independent of variations of surface, which leave the magnitude of the body unaltered. In conformity with this observation, it will be seen that at high velocities the thermal effect was nearly reduced to the quantity observed with the uncovered bulb. Similar remarks apply to the following results obtained after wrapping round the bulb a fine spiral of thin brass wire.

TABLE VII. - Bulb wrapped with a spiral of fine brass wire.

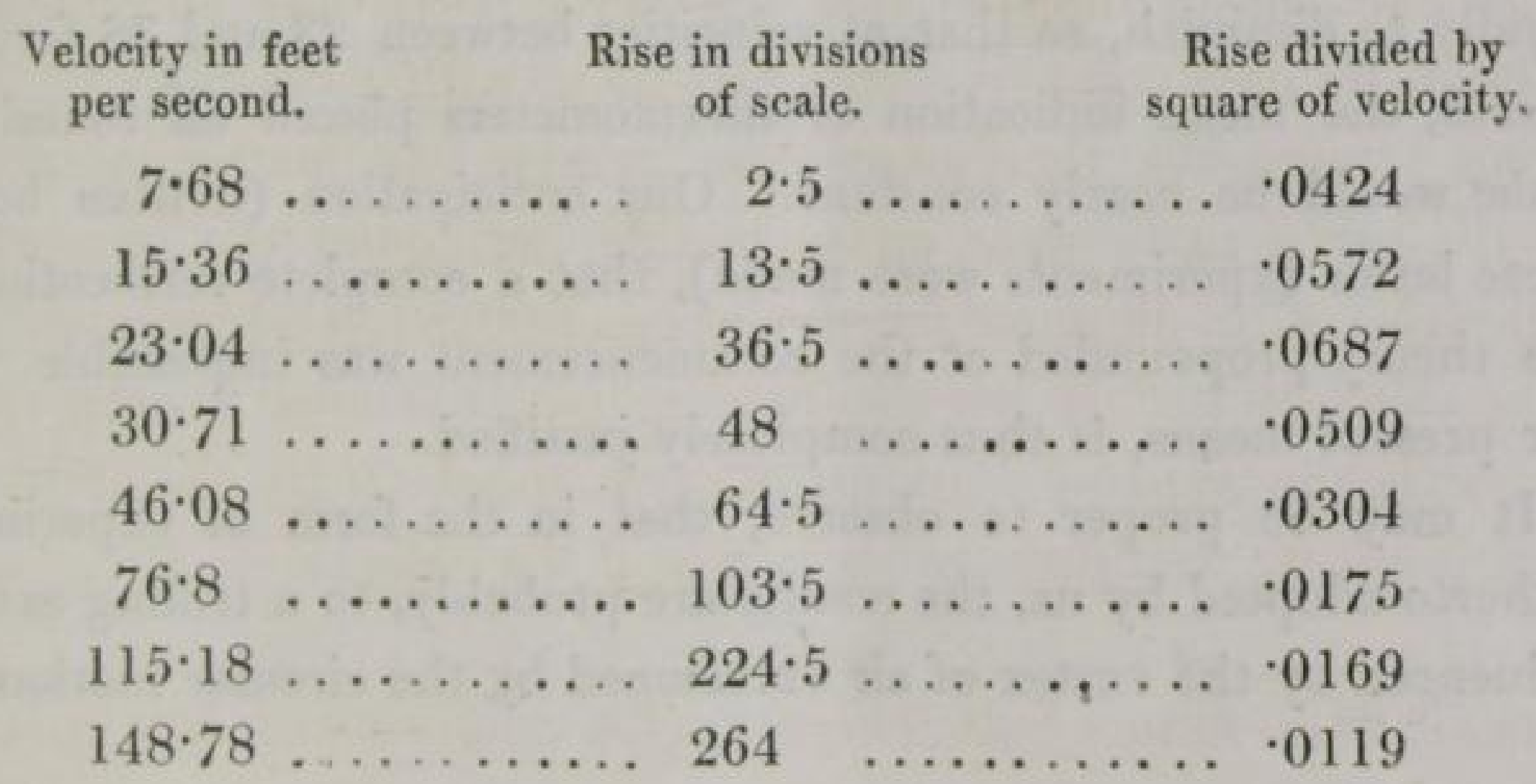

The thermal effects on different sides of a sphere moving through air, have been investigated by us experimentally by whirling a thin glass globe of 3.58 inches diameter along with the smaller thermometer, the bulb of which was placed successively in three positions, viz. in front, at one side, and in the rear. In each situation it was placed as near the glass globe as possible without actually touching it.

thermal effect must necessarily suffer diminution; unless indeed it gives rise to increased resistance. 
TABLE VIII.-Smaller Thermometer whirled along with glass globe.

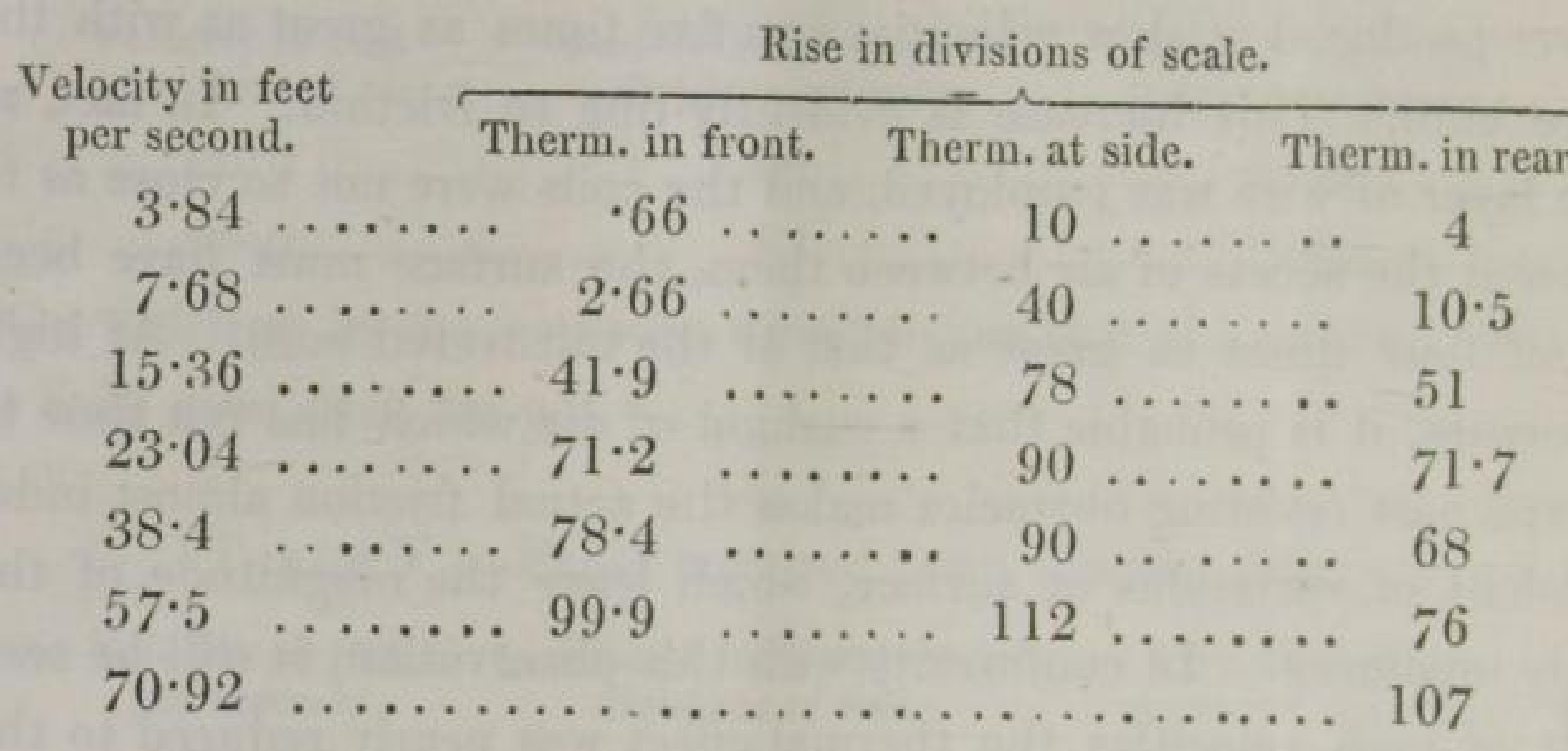

The effects of fluid friction are strikingly evident in the above results, particularly at the slow velocities of 3 and 7 feet per second. It is clear from these, that the air, after coming in contact with the front of the globe, traverses with friction the equatorial parts, giving out an accumulating thermal effect, a part of which is carried round to the after pole. At higher velocities the effects of friction seem rapidly to diminish, so that at velocities between 23 and 38 feet per second, the mean indication of thermometers placed all round the globe would be nearly constant. Our anticipation (written before these latter experiments were made), that a complete verification of the theory propounded at the commencement was impossible with our present means, is thus completely justified.

It may be proper to observe, that in the form of experiment hitherto adopted by us, the results are probably, to a trifling extent, influenced by the vortex of air occasioned by the circular motion.

We have on several occasions noticed the effect of sudden changes in the force of wind on the temperature of a thermometer held in it. Sometimes the thermometer was observed to rise, at other times to fall, when a gust came suddenly on. When a rise occurred, it was seldom equivalent to the effect, as ascertained by the foregoing experiments, due to the increased velocity of the air. Hence we draw the conclusion, that the actual temperature of a gust of wind is lower than that of the subsequent lull. This is probably owing to the air in the latter case having had its vis viva converted into heat by 
collision with material objects. In fact we find that in sheltered situations, such for instance as one or two inches above a wall opposite to the wind, the thermometer indicates a higher temperature than it does when exposed to the blast. The question, which is one of great interest for meteorological science, has hitherto been only partially discussed by us, and for its complete solution will require a careful estimate of the temperature of the earth's surface, of the effects of radiation, \&c., and also a knowledge of the causes of gusts in different winds.

[From the Proceedings of the Royal Society of London for June 15, 1857.] 


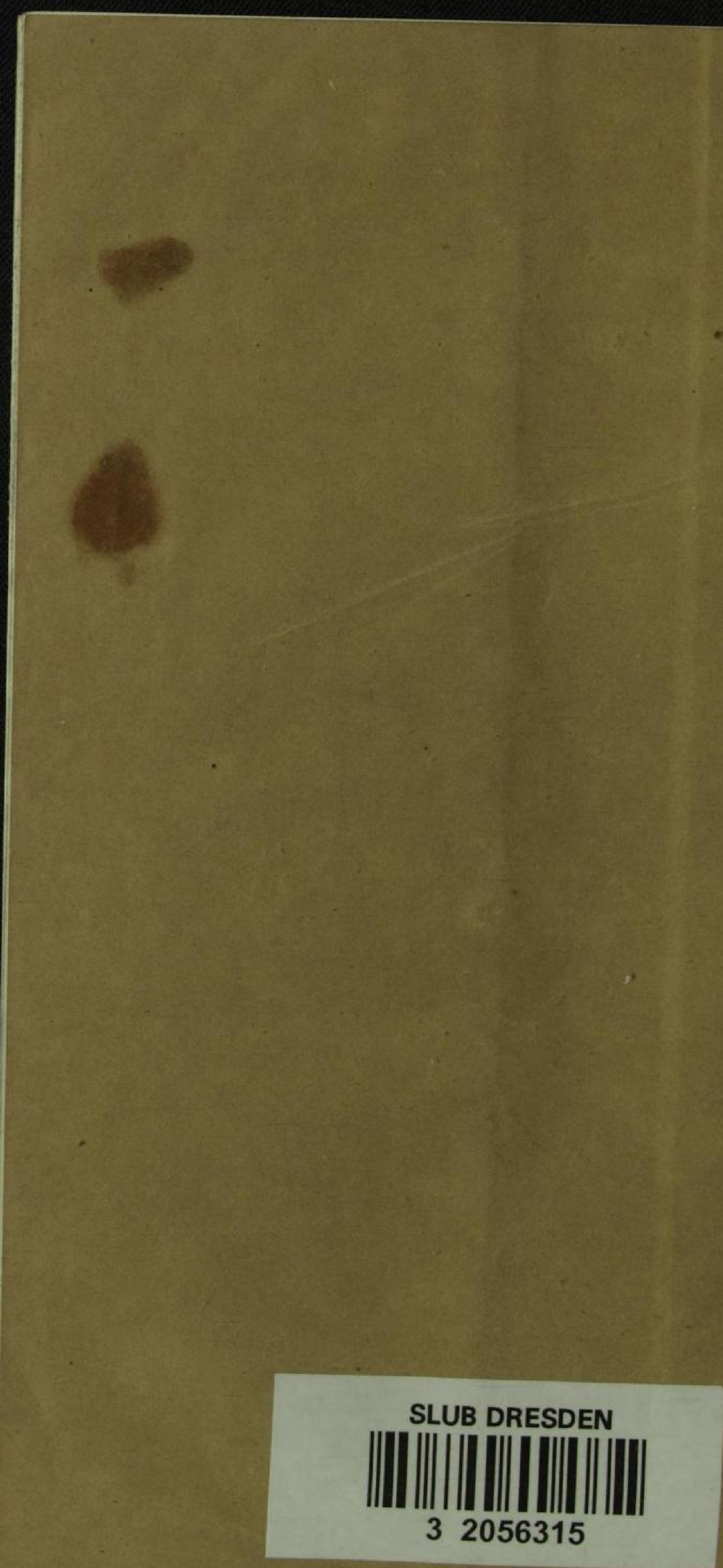

